The University of Southern Mississippi

The Aquila Digital Community

Faculty Publications

9-14-2009

\title{
A Spectral Time-Domain Method for Computational Electrodynamics
}

James V. Lambers

University of Southern Mississippi, james.lambers@usm.edu

Follow this and additional works at: https://aquila.usm.edu/fac_pubs

Part of the Chemistry Commons

\section{Recommended Citation}

Lambers, J. V. (2009). A Spectral Time-Domain Method for Computational Electrodynamics. AIP Conference Proceedings, 1168(1), 1-6.

Available at: https://aquila.usm.edu/fac_pubs/16212

This Article is brought to you for free and open access by The Aquila Digital Community. It has been accepted for inclusion in Faculty Publications by an authorized administrator of The Aquila Digital Community. For more information, please contact Joshua.Cromwell@usm.edu. 


\title{
A Spectral Time-Domain Method for Computational Electrodynamics
}

\author{
James V. Lambers *
}

\begin{abstract}
Ever since its introduction by Kane Yee over forty years ago, the finite-difference time-domain (FDTD) method has been a widely-used technique for solving the time-dependent Maxwell's equations. This paper presents an alternative approach to these equations in the case of spatially-varying electric permittivity and/or magnetic permeability, based on Krylov subspace spectral (KSS) methods. These methods have previously been applied to the variablecoefficient heat equation and wave equation, and have demonstrated high-order accuracy, as well as stability characteristic of implicit time-stepping schemes, even though KSS methods are explicit. KSS methods for scalar equations compute each Fourier coefficient of the solution using techniques developed by Gene Golub and Gérard Meurant for approximating elements of functions of matrices by Gaussian quadrature in the spectral, rather than physical, domain. We show how they can be generalized to coupled systems of equations, such as Maxwell's equations, by choosing appropriate basis functions that, while induced by this coupling, still allow efficient and robust computation of the Fourier coefficients of each spatial component of the electric and magnetic fields. We also discuss the implementation of appropriate boundary conditions for simulation on infinite computational domains, and how discontinuous coefficients can be handled.
\end{abstract}

Keywords: spectral methods, Gaussian quadrature, block Lanczos method, Maxwell's equations

\section{Introduction}

We consider Maxwell's equation on the rectangle $[0,2 \pi]^{3}$, with periodic boundary conditions. Assuming nonconductive material with no losses, we have

$$
\begin{gathered}
\operatorname{div} \hat{\mathbf{E}}=0, \quad \operatorname{div} \hat{\mathbf{H}}=0, \\
\operatorname{curl} \hat{\mathbf{E}}=-\mu \frac{\partial \hat{\mathbf{H}}}{\partial t}, \quad \operatorname{curl} \hat{\mathbf{H}}=\varepsilon \frac{\partial \hat{\mathbf{E}}}{\partial t},
\end{gathered}
$$

where $\hat{\mathbf{E}}, \hat{\mathbf{H}}$ are the vectors of the electric and magnetic fields, and $\varepsilon, \mu$ are the electric permittivity and magnetic permeability, respectively. We assume that these two functions are smoothly varying in space.

\footnotetext{
*Submitted December 30, 2008. Stanford University, Department of Energy Resources Engineering, Stanford, CA 94305-2220 USA Tel/Fax: 650-725-2729/2099 Email: lambers@stanford.edu
}

By taking the curl of both sides of (2), we decouple the vector fields $\hat{\mathbf{E}}$ and $\hat{\mathbf{H}}$ and obtain the equations

$$
\begin{aligned}
\mu \varepsilon \frac{\partial^{2} \hat{\mathbf{E}}}{\partial t^{2}} & =\Delta \hat{\mathbf{E}}+\mu^{-1} \operatorname{curl} \hat{\mathbf{E}} \times \nabla \mu, \\
\mu \varepsilon \frac{\partial^{2} \hat{\mathbf{H}}}{\partial t^{2}} & =\Delta \hat{\mathbf{H}}+\varepsilon^{-1} \operatorname{curl} \hat{\mathbf{H}} \times \nabla \varepsilon .
\end{aligned}
$$

In his 1966 paper [23], Yee proposed the original finitedifference time-domain method for solving the equations (1), (2). This method uses a staggered grid to avoid solving simultaneous equations for $\hat{\mathbf{E}}$ and $\hat{\mathbf{H}}$, and also removes numerical dissipation. However, because it is an explicit finite-difference scheme, its time step is constrained by the CFL condition. Nonetheless, it remains a widely used method to this day, and has inspired a host of related methods, including, for example, several that are based on spatial discretizations other than finite differences, such as a pseudospectral time-domain (PSTD) method [17], an FDTD-FEM hybrid method [19], and a one-step algorithm based on Chebyshev polynomial approximations [4]. In this paper, we introduce an new time-domain method for these equations.

In [15] a class of methods, called Krylov subspace spectral (KSS) methods, was introduced for the purpose of solving parabolic variable-coefficient PDE. These methods are based on techniques developed by Golub and Meurant in [5] for approximating elements of a function of a matrix by Gaussian quadrature in the spectral domain. In $[9,11]$, these methods were generalized to the second-orer wave equation, for which these methods have exhibited even higher-order accuracy.

It has been shown in these references that KSS methods, by employing different approximations of the solution operator for each Fourier component of the solution, achieve higher-order accuracy in time than other Krylov subspace methods (see, for example, [10]) for stiff systems of ODE, and, as shown in [11], they are also quite stable, considering that they are explicit methods. In $[12,13]$, the accuracy and robustness of KSS methods were enhanced using block Gaussian quadrature.

It is our hope that the high-order accuracy achieved for the scalar wave equation can be extended to systems of coupled wave equations such as those described by 
Maxwell's equations. Section 2 reviews the main properties of KSS methods, including block KSS methods, as applied to the parabolic problems for which they were originally designed. Section 3 reviews their application to the wave equation, including previous convergence analysis. In Section 4, we discuss the modifications that must be made to block KSS methods in order to apply them to Maxwell's equations, as well as issues that must be addressed in future work in order to obtain effective algorithms for solving more realistic problems involving these equations. Numerical results are presented in Section 5, and conclusions are stated in Section 6 .

\section{Krylov Subspace Spectral Methods}

We first review KSS methods, which are easier to describe for parabolic problems. Let $S(t)=\exp [-L t]$ represent the exact solution operator of the problem

$$
\begin{gathered}
u_{t}+L u=0, \quad t>0, \\
u(x, 0)=f(x), \quad 0<x<2 \pi, \\
u(0, t)=u(2 \pi, t), \quad t>0 .
\end{gathered}
$$

The operator $L$ is a second-order differential operator of the form

$$
L u=-\left(p(x) u_{x}\right)_{x}+q(x) u,
$$

where $p(x)$ is a positive function and $q(x)$ is a nonnegative (but nonzero) smooth function. It follows that $L$ is selfadjoint and positive definite.

Let $\langle\cdot, \cdot\rangle$ denote the standard inner product of functions defined on $[0,2 \pi]$,

$$
\langle f(x), g(x)\rangle=\int_{0}^{2 \pi} \overline{f(x)} g(x) d x .
$$

Krylov subspace spectral methods, introduced in [15], use Gaussian quadrature on the spectral domain to compute the Fourier components of the solution. These methods are time-stepping algorithms that compute the solution at time $t_{1}, t_{2}, \ldots$, where $t_{n}=n \Delta t$ for some choice of $\Delta t$. Given the computed solution $\tilde{u}\left(x, t_{n}\right)$ at time $t_{n}$, the solution at time $t_{n+1}$ is computed by approximating the Fourier components that would be obtained by applying the exact solution operator to $\tilde{u}\left(x, t_{n}\right)$,

$$
\hat{u}\left(\omega, t_{n+1}\right)=\left\langle\frac{1}{\sqrt{2 \pi}} e^{i \omega x}, S(\Delta t) \tilde{u}\left(x, t_{n}\right)\right\rangle .
$$

Krylov subspace spectral methods approximate these components with higher-order temporal accuracy than traditional spectral methods and time-stepping schemes.

\subsection{Elements of Functions of Matrices}

In [5] Golub and Meurant describe a method for computing quantities of the form

$$
\mathbf{u}^{T} f(A) \mathbf{v}
$$

where $\mathbf{u}$ and $\mathbf{v}$ are $N$-vectors, $A$ is an $N \times N$ symmetric positive definite matrix, and $f$ is a smooth function. Our goal is to apply this method with $A=L_{N}$ where $L_{N}$ is a spectral discretization of $L, f(\lambda)=\exp (-\lambda t)$ for some $t$, and the vectors $\mathbf{u}$ and $\mathbf{v}$ are derived from $\hat{\mathbf{e}}_{\omega}$ and $\mathbf{u}^{n}$, where $\hat{\mathbf{e}}_{\omega}$ is a discretization of $\frac{1}{\sqrt{2 \pi}} e^{i \omega x}$ and $\mathbf{u}^{n}$ is the approximate solution at time $t_{n}$, evaluated on an $N$-point uniform grid.

The basic idea is as follows: since the matrix $A$ is symmetric positive definite, it has real eigenvalues

$$
b=\lambda_{1} \geq \lambda_{2} \geq \cdots \geq \lambda_{N}=a>0,
$$

and corresponding orthogonal eigenvectors $\mathbf{q}_{j}, j=$ $1, \ldots, N$. Therefore, the quantity (11) can be rewritten as

$$
\mathbf{u}^{T} f(A) \mathbf{v}=\sum_{j=1}^{N} f\left(\lambda_{j}\right) \mathbf{u}^{T} \mathbf{q}_{j} \mathbf{q}_{j}^{T} \mathbf{v} .
$$

We let $a=\lambda_{N}$ be the smallest eigenvalue, $b=\lambda_{1}$ be the largest eigenvalue, and define the measure $\alpha(\lambda)$ by

$$
\alpha(\lambda)= \begin{cases}0, & \text { if } \lambda<a \\ \sum_{j=i}^{N} \alpha_{j} \beta_{j}, & \text { if } \lambda_{i} \leq \lambda<\lambda_{i-1} \\ \sum_{j=1}^{N} \alpha_{j} \beta_{j}, & \text { if } b \leq \lambda\end{cases}
$$

where $\alpha_{j}=\mathbf{u}^{T} \mathbf{q}_{j}$ and $\beta_{j}=\mathbf{q}_{j}^{T} \mathbf{v}$. If this measure is positive and increasing, then the quantity (11) can be viewed as a Riemann-Stieltjes integral

$$
\mathbf{u}^{T} f(A) \mathbf{v}=I[f]=\int_{a}^{b} f(\lambda) d \alpha(\lambda) .
$$

As discussed in [5], the integral $I[f]$ can be approximated using Gaussian quadrature rules, which yield an approximation of the form

$$
I[f]=\sum_{j=1}^{K} w_{j} f\left(t_{j}\right)+R[f],
$$

where the nodes $t_{j}, j=1, \ldots, K$, as well as the weights $w_{j}, j=1, \ldots, K$, can be obtained using the symmetric Lanczos algorithm if $\mathbf{u}=\mathbf{v}$, and the unsymmetric Lanczos algorithm if $\mathbf{u} \neq \mathbf{v}$ ( see [8]).

\section{$2.2 \quad$ Block Gaussian Quadrature}

In the case $\mathbf{u} \neq \mathbf{v}$, there is the possibility that the weights may not be positive, which destabilizes the quadrature rule (see [1] for details). One option to get around this problem is rewriting (11) using decompositions such as

$$
\mathbf{u}^{T} f(A) \mathbf{v}=\frac{1}{\delta}\left[\mathbf{u}^{T} f(A)(\mathbf{u}+\delta \mathbf{v})-\mathbf{u}^{T} f(A) \mathbf{u}\right],
$$

where $\delta$ is a small constant. Guidelines for choosing an appropriate value for $\delta$ can be found in [15, Section 2.2]. 
If we compute (11) using (17) or the polar decomposition

$$
\frac{1}{4}\left[(\mathbf{u}+\mathbf{v})^{T} f(A)(\mathbf{u}+\mathbf{v})-(\mathbf{v}-\mathbf{u})^{T} f(A)(\mathbf{v}-\mathbf{u})\right],
$$

then we could use the symmetric Lanczos algorithm, but we would still have to carry out the process for approximating an expression of the form (11) with two starting vectors. Instead, we consider

$$
\left[\begin{array}{ll}
\mathbf{u} & \mathbf{v}
\end{array}\right]^{T} f(A)\left[\begin{array}{ll}
\mathbf{u} & \mathbf{v}
\end{array}\right]
$$

which results in the $2 \times 2$ matrix

$$
\int_{a}^{b} f(\lambda) d \mu(\lambda)=\left[\begin{array}{ll}
\mathbf{u}^{T} f(A) \mathbf{u} & \mathbf{u}^{T} f(A) \mathbf{v} \\
\mathbf{v}^{T} f(A) \mathbf{u} & \mathbf{v}^{T} f(A) \mathbf{v}
\end{array}\right]
$$

where $\mu(\lambda)$ is a $2 \times 2$ matrix function of $\lambda$, each entry of which is a measure of the form $\alpha(\lambda)$ from (14).

In [5] Golub and Meurant show how a block method can be used to generate quadrature formulas. We will describe this process here in more detail. The integral $\int_{a}^{b} f(\lambda) d \mu(\lambda)$ is now a $2 \times 2$ symmetric matrix and the most general $K$-node quadrature formula is of the form

$$
\int_{a}^{b} f(\lambda) d \mu(\lambda)=\sum_{j=1}^{K} W_{j} f\left(T_{j}\right) W_{j}+\text { error }
$$

with $T_{j}$ and $W_{j}$ being symmetric $2 \times 2$ matrices. By diagonalizing each $T_{j}$, we obtain the simpler formula

$$
\int_{a}^{b} f(\lambda) d \mu(\lambda)=\sum_{j=1}^{2 K} f\left(\lambda_{j}\right) \mathbf{v}_{j} \mathbf{v}_{j}^{T}+\text { error }
$$

where, for each $j, \lambda_{j}$ is a scalar and $\mathbf{v}_{j}$ is a 2 -vector.

Each node $\lambda_{j}$ is an eigenvalue of the matrix

$$
\mathcal{T}_{K}=\left[\begin{array}{ccccc}
M_{1} & B_{1}^{T} & & & \\
B_{1} & M_{2} & B_{2}^{T} & & \\
& \ddots & \ddots & \ddots & \\
& & B_{K-2} & M_{K-1} & B_{K-1}^{T} \\
& & & B_{K-1} & M_{K}
\end{array}\right]
$$

which is a block-triangular matrix of order $2 K$. The vector $\mathbf{v}_{j}$ consists of the first two elements of the corresponding normalized eigenvector.

To compute the matrices $M_{j}$ and $B_{j}$, we use the block Lanczos algorithm, which was proposed by Golub and Underwood in [7]. Let $X_{0}$ be an $N \times 2$ given matrix, such that $X_{1}^{T} X_{1}=I_{2}$. Let $X_{0}=0$ be an $N \times 2$ matrix. Then, for $j=1, \ldots, K$, we compute

$$
\begin{gathered}
M_{j}=X_{j}^{T} A X_{j} \\
R_{j}=A X_{j}-X_{j} M_{j}-X_{j-1} B_{j-1}^{T} \\
X_{j+1} B_{j}=R_{j} .
\end{gathered}
$$

The last step of the algorithm is the $Q R$ decomposition of $R_{j}$ such that $X_{j+1}$ is $n \times 2$ with $X_{j+1}^{T} X_{j+1}=I_{2}$. The matrix $B_{j}$ is $2 \times 2$ and upper triangular. The other coefficient matrix $M_{j}$ is $2 \times 2$ and symmetric.

\subsection{Block KSS Methods}

We are now ready to describe block KSS methods. For each wave number $\omega=-N / 2+1, \ldots, N / 2$, we define

$$
R_{0}(\omega)=\left[\begin{array}{ll}
\hat{\mathbf{e}}_{\omega} & \mathbf{u}^{n}
\end{array}\right]
$$

and compute the $Q R$ factorization $R_{0}(\omega)=X_{1}(\omega) B_{0}(\omega)$. We then carry out the block Lanczos iteration described in (23) to obtain a block tridiagonal matrix $\mathcal{T}_{K}(\omega)$ of the form (22), where each entry is a function of $\omega$.

Then, we can express each Fourier component of the approximate solution at the next time step as

$$
\left[\hat{\mathbf{u}}^{n+1}\right]_{\omega}=\left[B_{0}^{H} E_{12}^{H} \exp \left[-\mathcal{T}_{K}(\omega) \Delta t\right] E_{12} B_{0}\right]_{12}
$$

where $E_{12}=\left[\begin{array}{ll}\mathbf{e}_{1} & \mathbf{e}_{2}\end{array}\right]$. The computation of (24) consists of computing the eigenvalues and eigenvectors of $\mathcal{T}_{K}(\omega)$ in order to obtain the nodes and weights for Gaussian quadrature, as described earlier.

This algorithm has local temporal accuracy $O\left(\Delta t^{2 K}\right)$ [12]. Furthermore, block KSS methods are significantly more accurate than the original KSS methods described in $[15,11]$, that employ either (17) and (18), even though they have the same temporal order of accuracy, because the solution plays a greater role in the determination of the quadrature nodes. They are also more effective for problems with oscillatory or discontinuous coefficients.

\section{Application to the Wave Equation}

In this section we review the application of Krylov subspace spectral methods to the problem

$$
\begin{gathered}
u_{t t}+L u=0 \quad \text { on }(0,2 \pi) \times(0, \infty), \\
u(x, 0)=f(x), \quad u_{t}(x, 0)=g(x), \quad 0<x<2 \pi,
\end{gathered}
$$

with periodic boundary conditions

$$
u(0, t)=u(2 \pi, t), \quad t>0 .
$$

A spectral representation of the operator $L$ allows us the obtain a representation of the solution operator (the propagator) in terms of the sine and cosine families generated by $L$ by a simple functional calculus. Introduce

$$
\begin{aligned}
& R_{1}(t)=L^{-1 / 2} \sin (t \sqrt{L})=\sum_{n=1}^{\infty} \frac{\sin \left(t \sqrt{\lambda_{n}}\right)}{\sqrt{\lambda_{n}}}\left\langle\varphi_{n}^{*}, \cdot\right\rangle \varphi_{n} \\
& R_{0}(t)=\cos (t \sqrt{L})=\sum_{n=1}^{\infty} \cos \left(t \sqrt{\lambda_{n}}\right)\left\langle\varphi_{n}^{*}, \cdot\right\rangle \varphi_{n}
\end{aligned}
$$

where $\lambda_{1}, \lambda_{2}, \ldots$ are the (positive) eigenvalues of $L$, and $\varphi_{1}, \varphi_{2}, \ldots$ are the corresponding eigenfunctions. Then the propagator of (25) can be written as

$$
P(t)=\left[\begin{array}{cc}
R_{0}(t) & R_{1}(t) \\
-L R_{1}(t) & R_{0}(t)
\end{array}\right]
$$


The entries of this matrix, as functions of $L$, indicate which functions are the integrands in the RiemannStieltjes integrals used to compute the Fourier components of the solution.

Block KSS methods can be applied to the wave equation in the same way as for parabolic problems, as described in Section 2.3, except that the block Lanczos algorithm is used twice for each Fourier coefficient, to compute the solution and its time derivative.

We now review the convergence analysis of block KSS methods carried out in [13].

Theorem 1 Let $L$ be a self-adjoint 2nd-order positive definite differential operator on $C_{p}([0,2 \pi])$ with coefficients in $B L_{M}([0,2 \pi])$ for a fixed integer $M$, and let $f, g \in C_{p}^{n}([0,2 \pi])$ for $n \geq 4 K$ for a positive integer $K$. Let $N \geq M$, and that for each $\omega=-N / 2+1, \ldots, N / 2$, the recursion coefficients in (22) are computed on a $2^{K} N$ point uniform grid. Then a block KSS method that uses a K-node block Gaussian rule to compute each Fourier component $\left[\hat{\mathbf{u}}^{1}\right]_{\omega}$, for $\omega=-N / 2+1, \ldots, N / 2$, of the solution to (25), (26), (27), and each Fourier component $\left[\hat{\mathbf{u}}_{t}^{1}\right]_{\omega}$ of its time derivative, satisfies

$$
\begin{gathered}
\left|\left[\hat{\mathbf{u}}^{1}\right]_{\omega}-\hat{u}(\omega, \Delta t)\right|=O\left(\Delta t^{4 K}\right), \\
\left|\left[\hat{\mathbf{u}}_{t}^{1}\right]_{\omega}-\hat{u}_{t}(\omega, \Delta t)\right|=O\left(\Delta t^{4 K-1}\right),
\end{gathered}
$$

where $\hat{u}(\omega, \Delta t)$ is the corresponding Fourier component of the exact solution at time $\Delta t$.

\section{Proof. See [13, Theorem 5].}

In $[13$, Theorem 6$]$, it is shown that when the leading coefficient $p(x)$ is constant and the coefficient $q(x)$ is bandlimited, the 1-node KSS method, which has third-order local accuracy in time, is also unconditionally stable. This result, and Theorem 1, imply convergence for the 1-node method, with second-order global temporal accuracy.

\section{Application to Maxwell's Equations}

In this section, we consider the various generalizations that must be made to block KSS methods for the wave equation in order to apply them to Maxwell's equations, and then discuss the performance of the resulting algorithm.

\subsection{Generalization to Systems of Equations}

First, we consider the following initial-boundary value problem in one space dimension,

$$
\begin{gathered}
\frac{\partial^{2} \mathbf{u}}{\partial t^{2}}+L \mathbf{u}=0, \quad t>0, \\
\mathbf{u}(x, 0)=\mathbf{f}(x), \quad \frac{\partial \mathbf{u}}{\partial t}(x, 0)=\mathbf{g}(x), \quad 0<x<2 \pi,
\end{gathered}
$$

with periodic boundary conditions

$$
\mathbf{u}(0, t)=\mathbf{u}(2 \pi, t), \quad t>0,
$$

where $\mathbf{u}:[0,2 \pi] \times[0, \infty) \rightarrow \mathbb{R}^{n}$ for $n>1$, and $L(x, D)$ is an $n \times n$ matrix where the $(i, j)$ entry is an a differential operator $L_{i j}(x, D)$ of the form

$$
L_{i j}(x, D) u(x)=\sum_{\mu=0}^{m_{i j}} a_{\mu}^{i j}(x) D^{\mu} u, \quad D=\frac{d}{d x},
$$

with spatially varying coefficients $a_{\mu}^{i j}, \mu=0,1, \ldots, m_{i j}$.

Generalization of KSS methods to a system of the form (31) can proceed as follows. For $i, j=1, \ldots, n$, let $\bar{L}_{i j}(D)$ be the constant-coefficient operator obtained by averaging the coefficients of $L_{i j}(x, D)$ over $[0,2 \pi]$. Then, for each wave number $\omega$, we define $L(\omega)$ be the matrix with entries $\bar{L}_{i j}(\omega)$, i.e., the symbols of $\bar{L}_{i j}(D)$ evaluated at $\omega$. Next, we compute the spectral decomposition of $L(\omega)$ for each $\omega$. For $j=1, \ldots, n$, let $\mathbf{q}_{j}(\omega)$ be the left eigenvectors of $L(\omega)$. Then, we define our test functions by $\mathbf{q}_{j}(\omega) \otimes e^{i \omega x}$, and the trial functions are defined similarly using the right eigenvectors.

The recursion coefficients, nodes and weights can be computed in the same manner as in the scalar, self-adjoint case, with obvious modifications to account for the fact that the matrix $T_{\omega}\left(\delta_{\omega}\right)$, for each $\omega$, is no longer Hermitian. Once the components of the solution in our basis of trial functions is computed, the Fourier coefficients of each component function can be computed by solving $n N$ linear systems of size $n \times n$.

\subsection{Discontinuous Coefficients and Data}

As shown in $[16,13]$, rough or discontinuous coefficients reduce the accuracy of KSS methods, because they introduce significant spatial discretization error into the computation of recursion coefficients.

Ongoing work, described in [14], involves the use of the polar decomposition (18), to alleviate difficulties caused by such coefficients and initial data. This approach uses symmetric perturbations of initial Lanczos vectors in the direction of the solution in order to cancel out highfrequency oscillations. Future work will explore possible combinations of this approach with block KSS methods in order to generalize the superior accuracy of the block approach to these more difficult problems.

Alternatively, adaptive spatial resolution has been shown to be effective for handling multilayer profiles in TE and TM polarizations (see [22]), which KSS methods can readily incorporate as well.

\subsection{Other Boundary Conditions}

While we have used periodic boundary conditions in this paper, it is typical in practical applications to use bound- 
ary conditions that are more effective at simulating an infinite domain. One such type of boundary condition is a perfectly matched layer (PML), first used by Berenger in [2] for Maxwell's equations. A PML absorbs waves by modifying spatial differentiation operators in the PDE. For example, for absorbing waves that propagate in the $x$ direction, $\frac{\partial}{\partial x}$ is replaced by $\frac{1}{1+\frac{i \sigma(x)}{\omega}} \frac{\partial}{\partial x}$, where, as before, $\omega$ represents the wave number, and $\sigma$ is a positive function that causes propagating waves to be attenuated.

In KSS methods, this transformation can be incorporated into the symbol of the operator $L$ when computing the recursion coefficients. The dependence of the transformation on both $x$ and $\omega$ makes the efficient application of the transformed operator more difficult, especially in higher space dimensions, but recent work on rapid application of Fourier integral operators (see [3]) can mitigate this concern. Future work will explore the use of PML, taking into account very recent analysis in [18] of the difficulties of PML with inhomogeneous media, and the remediation of these difficulties through adiabatic absorbers.

\subsection{Accuracy}

Let $A$ be a symmetric matrix with eigenvalues (12). The error $R[f]$ in the approximation of $\mathbf{u}^{T} f(A) \mathbf{v}$ by a quadrature rule of the form (21) is given by

$$
R[f]=\frac{1}{(2 K) !} \int_{a}^{b} \frac{d^{2 K} f(\xi(\lambda))}{d \lambda^{2 K}} \prod_{j=1}^{2 K}\left(\lambda-\lambda_{j}\right) d \alpha(\lambda),
$$

where $\alpha(\lambda)$ is as defined in (14). In this case, the matrix $A_{N}$ that discretizes the operator

$$
A \hat{\mathbf{E}}=\frac{1}{\mu \varepsilon}\left(\Delta \hat{\mathbf{E}}+\mu^{-1} \operatorname{curl} \hat{\mathbf{E}} \times \nabla \mu\right)
$$

is not symmetric, and for each component of the solution, the resulting quadrature nodes $t_{j}, j=1, \ldots, 2 K$, are complex. In this case, the integral (35) is defined on a contour in the complex plane that passes through the eigenvalues of $A$, as discussed in [20]. Future work will include detailed analysis of the quadrature error, but what we can readily observe is that the dependence of this error on $\Delta t$ is the same as in the symmetric case, which bodes well for application to Maxwell's equations.

\section{$5 \quad$ Numerical Results}

We now apply a 2-node block KSS method to the equation (3), with initial conditions

$$
\hat{\mathbf{E}}(x, y, z, 0)=\mathbf{F}(x, y, z), \quad \frac{\partial \hat{\mathbf{E}}}{\partial t}(x, y, z, 0)=\mathbf{G}(x, y, z),
$$

with periodic boundary conditions. The coefficients $\mu$ and $\varepsilon$ are constructed from randomly generated, damped
Fourier coefficients as described in [15]. Specifically,

$$
\begin{aligned}
\mu(x, y, z) \approx & 0.4077+0.0039 \cos z+0.0043 \cos y- \\
& 0.0012 \sin y+0.0018 \cos (y+z)+ \\
& 0.0027 \cos (y-z)+0.003 \cos x+ \\
& 0.0013 \cos (x-z)+0.0012 \sin (x-z)+ \\
& 0.0017 \cos (x+y)+0.0014 \cos (x-y), \\
\varepsilon(x, y, z) \approx & 0.4065+0.0025 \cos z+0.0042 \cos y+ \\
& 0.001 \cos (y+z)+0.0017 \cos x+ \\
& 0.0011 \cos (x-z)+0.0018 \cos (x+y)+ \\
& 0.002 \cos (x-y) .
\end{aligned}
$$

The components of $\mathbf{F}$ and $\mathbf{G}$ are generated in a similar fashion, except that the $x$ - and $z$-components are zero.

Table 1 lists error estimates for solutions computed using $K=2$ block quadrature nodes per component in the basis described in Section 4.1. The error estimate for each solution is obtained by taking the $\ell_{2}$-norm of the relative difference between the solution, and a solution computed using a smaller time step $\Delta t=1 / 64$.

We observe that as the number of grid points increases, the temporal order of accuracy also increases toward the theoretical expectation of 6th-order accuracy, due to the reduced spatial error arising from the truncation of Fourier series. Also, increasing the resolution does not pose any difficulty from a stability point of view; for a fixed time step, accuracy increases with resolution.

\begin{tabular}{|r|r|r|}
\hline$\Delta t$ & $N=16$, Order $=5.52$ & $N=32$, Order $=5.84$ \\
\hline 1 & $6.654 \mathrm{e}-3$ & $3.641 \mathrm{e}-3$ \\
$1 / 2$ & $2.577 \mathrm{e}-4$ & $7.466 \mathrm{e}-5$ \\
$1 / 4$ & $3.233 \mathrm{e}-6$ & $8.301 \mathrm{e}-7$ \\
$1 / 8$ & $4.888 \mathrm{e}-8$ & $9.432 \mathrm{e}-9$ \\
$1 / 16$ & $7.656 \mathrm{e}-10$ & $1.571 \mathrm{e}-10$ \\
$1 / 32$ & $3.264 \mathrm{e}-11$ & $5.922 \mathrm{e}-12$ \\
\hline
\end{tabular}

Table 1: Estimates of relative error in solutions of (3), (36) computed using a 2-node block KSS method on an $N$-point grid, with time step $\Delta t$, for various values of $N$ and $\Delta t$. For each $N$, the order of convergence is measured using the error estimates from time steps 1 and $1 / 32$.

We tried solving this same problem with MATLAB's most accurate ODE solvers, ode45 and ode15s, the algorithms for which are described in [21]. Unfortunately, ode15s used too much memory. Accurate results could be obtained for ode 45 for the coarser grid, and these were comparable to the results obtained by KSS methods, although the order of accuracy was slightly less. However, as $N$ increased, ode 45 was unable to obtain reasonable accuracy for the same time steps, due to instability. 


\section{Conclusions}

We have demonstrated that KSS methods can be applied to Maxwell's equations with smoothly varying coefficients. The temporal accuracy is the same as for the wave equation, even though Fourier components are now represented by bilinear forms involving non-self-adjoint matrices, which are treated as Riemann-Stieltjes integrals over contours in the complex plane. Future work will extend the approach described in this paper to more realistic applications by using symbol modification to efficiently implement perfectly matched layers, and various techniques to effectively handle discontinuous coefficients.

\section{Acknowledgments}

The author thanks John J. Hench and Zdeněk Strakoš for the introduction to computational electrodynamics, as well helpful and stimulating discussions.

\section{References}

[1] Atkinson, K.: An Introduction to Numerical Analysis, 2nd Ed. Wiley (1989)

[2] Berenger, J,: A perfectly matched layer for the absorption of electromagnetic waves. J. Comp. Phys. 114 (1994) 185-200.

[3] Candes, E., Demanet, L., Ying, L.: Fast Computation of Fourier Integral Operators. SIAM J. Sci. Comput. 29(6) (2007) 2464-2493.

[4] De Raedt, H., Michielsen, K., Kole, J. S., Figge, M. T.: Solving the Maxwell equations by the Chebyshev method: A one-step finite difference timedomain algorithm. Ant. Prop., IEEE Trans. 51 (2003) 3155-3160.

[5] Golub, G. H., Meurant, G.: Matrices, Moments and Quadrature. Proceedings of the 15th Dundee Conference, June-July 1993, Griffiths, D. F., Watson, G. A. (eds.), Longman Scientific \& Technical (1994)

[6] Golub, G. H., Gutknecht, M. H.: Modified Moments for Indefinite Weight Functions. Numerische Mathematik 57 (1989) 607-624.

[7] Golub, G. H., Underwood, R.: The block Lanczos method for computing eigenvalues. Mathematical Software III, J. Rice Ed., (1977) 361-377.

[8] Golub, G. H, Welsch, J.: Calculation of Gauss Quadrature Rules. Math. Comp. 23 (1969) 221-230.

[9] Guidotti, P., Lambers, J. V., Sølna, K.: Analysis of 1-D Wave Propagation in Inhomogeneous Media. Numerical Functional Analysis and Optimization $\mathbf{2 7}$ (2006) 25-55.
[10] Hochbruck, M., Lubich, C.: On Krylov Subspace Approximations to the Matrix Exponential Operator. SIAM Journal of Numerical Analysis 34 (1996) 1911-1925.

[11] Lambers, J. V.: Derivation of High-Order Spectral Methods for Time-dependent PDE using Modified Moments. Electronic Transactions on Numerical Analysis 28 (2008) 114-135.

[12] Lambers, J. V.: Enhancement of Krylov Subspace Spectral Methods by Block Lanczos Iteration. Electronic Transactions on Numerical Analysis $\mathbf{3 1}$ (2008) in press.

[13] Lambers, J. V.: An Explicit, Stable, High-Order Spectral Method for the Wave Equation Based on Block Gaussian Quadrature. IAENG Journal of Applied Mathematics 38 (2008) 333-348.

[14] Lambers, J. V.: Krylov Subspace Spectral Methods for the Time-Dependent Schrödinger Equation with Non-Smooth Potentials. Submitted.

[15] Lambers, J. V.: Krylov Subspace Spectral Methods for Variable-Coefficient Initial-Boundary Value Problems. Electronic Transactions on Numerical Analysis 20 (2005) 212-234.

[16] Lambers, J. V.: Practical Implementation of Krylov Subspace Spectral Methods. Journal of Scientific Computing 32 (2007) 449-476.

[17] Liu, Q. H.: The pseudospectral time-domain (PSTD) method: A new algorithm for solutions of Maxwell's equations. Ant. Prop. Soc. Int'l Symp. Dig., IEEE 1 (1997) 122-125.

[18] Oskooi, A. F., Zhang, L., Avniel, Y., and Johnson, S. G.: The failure of perfectly matched layers, and towards their redemption by adiabatic absorbers. Opt. Expr. 16 (2008) 11376-11392.

[19] Rylander, T., Bondeson, A.: Stable FDTD-FEM hybrid method for Maxwell's equations. Comp. Phys. Comm. 125 (2000) 75-82.

[20] Saylor, P. E., Smolarski, D. C.: Why Gaussian quadrature in the complex plane? Num. Alg. 26 (2001) 251-280.

[21] Shampine, L. F., Reichelt, M. W.: The MATLAB ODE suite. SIAM Journal of Scientific Computing 18 (1997) 1-22.

[22] Vallius, T., Honkanen, M.: Reformulation of the Fourier nodal method with adaptive spatial resolution: application to multilevel profiles. Opt. Expr. 10(1) (2002) 24-34.

[23] Yee, K.: Numerical solution of initial boundary value problems involving Maxwell's equations in isotropic media. Ant. Prop., IEEE Trans. 14 (1966) 302-307. 